\title{
X-RAY OBSERVATIONS OF CLASSICAL AND RECURRENT NOVAE IN OUTBURST
}

\author{
M. Orio ${ }^{1,2}$ \\ RESUMEN
}

Presento una revisión de las observaciones de rayos $\mathrm{X}$ de novas clásicas y recurrentes en explosión, algunas de las cuales (12 objetos) fueron hechas recientemente con Chandra y XMM-Newton. La envolvente de la nova emite un flujo significativo de rayos $X$, con luminosidad pico de hasta $L_{x}=10^{35} \mathrm{erg} \mathrm{s}^{-1}$ en el rango de $0.2-10$ $\mathrm{keV}$. En sistemas de novas recurrentes, o en novas que contienen una gigante roja, la fuente de rayos $\mathrm{X}$ puede ser materia circunestelar previa, sacudida por el viento de la nova. Sin embargo, para la mayoría de novas clásicas los rayos-X se originan dentro de la nebulosa que es arrojada durante la explosión. Los datos indican una alta fracción de material chocado y un flujo emergente de viento inhomogéneo y variable. También se observa un espectro de líneas de emisión nebulares en las fases tardías. En aproximadamente la mitad de las novas observadas la enana blanca central aparece como una fuente muy luminosa de rayos X supersuaves de 1 a 9 años después de la explosión. El mejor tipo de objeto para estudiar las características de la quema de hidrógeno de la envolvente son las enanas blancas en sistemas degenerados simples. Las estadísticas, hasta ahora incompletas, indican que la duración de la fase de rayos $\mathrm{X}$ supersuaves presenta el pico alrededor de $\simeq$ 2 años. La correlación entre la curva de luz de rayos X y las propiedades de las novas no está del todo clara. Recientemente se obtuvieron "patrones de espectros de rejilla" con alta señal-a-ruido para V4743 Sgr. La curva de luz de rayos $\mathrm{X}$ de esta nova revela un espectro de potencia rico y complejo, con huellas de oscilaciones en modo $g$ no radiales de la enana blanca. Las oscilaciones y los espectros nos permiten determinar las propiedades de la enana blanca con quema de hidrógeno de la envolvente.

\begin{abstract}
I review X-ray observations of classical and recurrent novae in outburst, some of them recently done with Chandra and XMM-Newton for 12 objects. Significant X-ray flux is emitted by the nova shell, with a peak luminosity up to $\mathrm{L}_{\mathrm{x}}=10^{35} \mathrm{erg} \mathrm{s}^{-1}$ in the $0.2-10 \mathrm{keV}$ range. In recurrent nova systems, or in novae hosting a red giant, the source of X-rays may be previous circumstellar matter shocked by the nova wind. However, for most classical novae, X-rays originate inside the nebula ejected in the outburst. The data indicate a very high fraction of shocked material, and a non-smooth, varying wind outflow. A nebular emission line spectrum is also observed at late phases. In about half of the observed novae, the central white dwarf appears as a very luminous supersoft X-ray source for 1 to 9 years after the outburst. It is the best type of object to study the characteristics of shell hydrogen burning on white dwarfs in single degenerate systems. Still incomplete statistics indicate that the duration of the supersoft $X$-ray phase is peaked around $\simeq 2$ years. The correlation of the X-ray light curve with the nova properties is not quite clear. Recently, "template grating spectra" with high S/N have been obtained for V4743 Sgr. The X-ray light curve of this nova reveals a rich and complex power spectrum, with signatures of non-radial g-mode oscillations of the white dwarf. The oscillations and the spectra allow to determine the properties of the shell hydrogen burning white dwarf.
\end{abstract}

\section{Key Words: BINARIES: CLOSE - WHITE DWARFS - X-RAYS: STARS}

\section{MECHANISMIS OF X-RAY EMISSION FROM NOVAE}

Classical and recurrent novae are an ancient topic in astronomy, traditionally studied by optical astronomers. Yet, they emit in all wavelengths from

\footnotetext{
${ }^{1}$ INAF - Turin Astronomical Observatory, Italy.

${ }^{2}$ Department of Astronomy, U Wisconsin at Madison, USA.
}

gamma to radio, and are also an interesting subject of study for the younger science of X-ray astronomy. Novae may emit $\mathrm{X}$-rays because of four different physical mechanisms: 1) Shocks in the ejected wind or between the ejecta and interstellar or circumstellar medium, which produce a thermal bremsstrahlung spectrum, and probably, later, ionize of the ejecta, 2) Thermal emission of the central white dwarf (hereafter WD) atmosphere, like in su- 
persoft X-ray sources (see Greiner 2000), 3) Resumed accretion (through a disk or magnetic) for which a thermal bremsstrahlung spectrum is also observed, (see Orio et al. 2001a and references therein) 4) Finally, X-ray flux may result from Compton degradation produced by radioactive decays (Livio et al. 1992). The third and fourth mechanism are very interesting to study the secular evolution of novae and their nucleosynthesis output, respectively. However, I will review here only the first two mechanisms, which are now generally known to occur in most novae. The emission originating from the nova shell teaches about the wind emission and the the nebular physics, indicating electron density, plasma temperatures, and conditions of clumping and asymmetry in the ejecta. The atmospheric emission from the central WD offers instead a unique possibility to derive the physical parameters of an extremely hot white dwarf which is burning hydrogen in a shell. A single-degenerate binary system hosting such a WD is a potential type Ia SN progenitor. It is still a matter of debate whether recurrent novae are statistically significant as type Ia SN progenitors, and we know that only few classical novae may be. However, post-outburst nova WD are of great interest for type Ia supernova studies, because we seldom have the possibility to observe the atmosphere of a WD in these extreme conditions. In other types of supersoft X-ray sources, the central WD seems to be often obscured by a wind outflow, or an extended disk corona (e.g. Cal 87 described by Greiner et al., and Orio et al., at this conference). Novae that appear as supersoft X-ray sources for long enough that their atmosphere is no longer totally or partially hidden by the ejecta, are therefore very important "templates" of shell hydrogen burning WD. In order to assess if the WD is increasing in mass after each outburst and the nova we are observing is a rare type Ia SN candidate, we need to verify that the luminous supersoft X-ray source is observed for a long enough time to imply a significant amount of hydrogen retained after each outburst, and that the chemical abundances do not indicate the origin of the burning material in the "eroded" WD interior, rather than in the accreted matter. However, some classical novae, and especially recurrent novae (like perhaps U Sco, see Anupama and Dewangan 2000, but also Iijima 2002), may also become type Ia $\mathrm{SN}$ via a sub-Chandra mechanism of explosion accumulating a thick helium buffer (see Livio 2003, this conference). Since we need to know the abundances in the burning layer in both cases, high $\mathrm{S} / \mathrm{N}$ grating observations are essential.

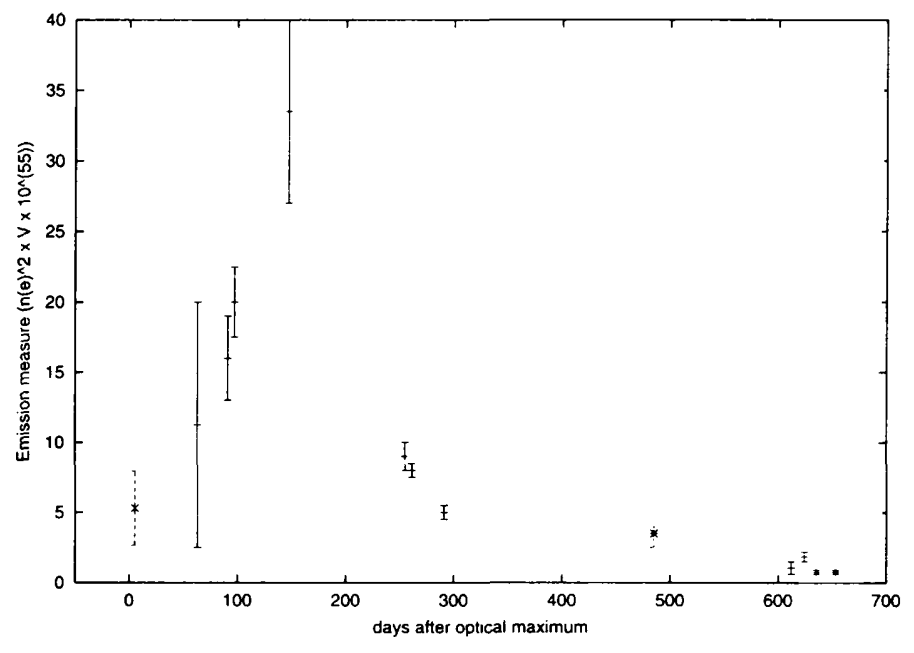

Fig. 1. The emission measure measured with ROSAT at different post-outburst epochs for V1974 Cyg (crosses), V838 Her (x), and V351 Pup (asterisk).

\section{THE EARLY OBSERVATIONS}

Novae in outburst were not pointed at with Einstein and previous X-ray satellites. NQ Vul and V1500 Cyg were serendipitously observed and not detected (a dubious 1- $\sigma$ detection is recorded for V1500 Cyg). Ögelman, Beuermann and Krautter $(1984,1987)$ used the Exosat LE telescope to observe three classical novae in outburst. An initial increase of X-ray flux was followed by a plateau and finally a decay. Despite poor $\mathrm{S} / \mathrm{N}$ and spectral resolution, the authors suggested that the X-ray emission was due to the central WD. The light curve of $\mathrm{RS} \mathrm{Oph}$ was also monitored with Exosat, but the X-rays emission was attributed instead to the nova wind colliding with circumstellar material, previously emitted in a wind by the red giant companion (Mason et al. 1987, Contini et al. 1995).

\section{THE HOT EJECTED SHELL}

Between 1989 and 1999 ROSAT was used to observe novae in the $0.2-2.4 \mathrm{keV}$ energy range, with moderate spectral resolution (with the PSPC) and 5 arcsec spatial resolution (with the HRI). ROSAT also contributed many serendipitous observations thanks to the 1 degree field of view of the PSPC and to the all-sky-survey it performed. 3 out of 7 Galactic and Magellanic Clouds novae were observed in the first 2 post-outburst years, and showed $\mathrm{X}$-ray emission of non-atmospheric origin, "hard" in the $R O S A T$ range. It was attributed to thermal emission in the ejected shell. 2 more novae seem to have been hard sources although they were only observed with the HRI (see Orio et al. 2001a for a discussion). 2-3 years after the outburst, no correlation was found between hard X-ray flux and post-outburst 
time. Probably only accreting sources were still observed. 4-6 years old novae were never detected, suggesting shorter cooling times of the shells. The novae observed with EXOSAT were also re-observed. GQ Mus was found to be still bright but only supersoft (Ögelman et al. 1993). PW Vul had faded, QU Vul was detected but appeared to have become much fainter and it was not supersoft (Orio et al. 2001a). Balman et al. (1998) fitted the spectrum of V1974 Cyg with a simple double model to disentangle the supersoft central source and the nebular component. They concluded that the X-ray luminosity was $\mathrm{L}(\mathrm{x})=10^{33-34} \mathrm{erg} \mathrm{s}^{-1}$ at maximum and it decreased in time, like the emission measure in Fig. 1. The high value of the emission measure $\left(\approx \mathrm{n}_{\mathrm{e}}^{2} \times \mathrm{V}\right)$, up to few $10^{56} \mathrm{~cm}^{-2}$ at maximum, indicated a large amount of shocked material. In Fig. 1 I plot the emission measure as a function of time including sporadic measurements for two other novae, V838 Her (Lloyd et al. 1992) and V351 Pup (Orio et al. 1996), that agree well with the V1494 Cyg curve.

After V1974 Cyg, a second optically bright nova became extremely bright in X-rays, V382 Vel, observed in 1999-2000 with four different satellites: Beppo-SAX, ASCA, Rossi-XTE and Chandra. This nova was a hard source at 15 days post-outburst, with a plasma temperature $\mathrm{kT} \approx 6 \mathrm{keV}$ (Orio et al. $2001 \mathrm{~b})$. The emission measure in the $0.2-10 \mathrm{keV}$ range was a factor 10 larger than observed for V1974 Cyg at maximum in the $0.2-2.4 \mathrm{keV}$ range. There was an initial rise in plasma temperature, then a slow decay in the next two months. A luminous supersoft component was predominant after 6 months (Orio et al. 2002), but not all the supersoft X-ray flux could due to the central WD. The spectrum could not be fitted with any atmospheric model. Four months later, only emission lines were observed in the supersoft X-ray range with the Chandra-LETG grating (Burwitz et al. 2002). Orio et al. (2002) concluded that the emission lines, of nebular origin, were already present and superimposed on the atmospheric continuum of the WD at the peak of the supersoft $\mathrm{X}$-ray phase, making it difficult to disentangle the atmospheric component. Mukai and Ishida (2001) analysed observations done in the first two months and measured a peak luminosity $\mathrm{L}(\mathrm{x}) \simeq 10^{35} \mathrm{erg} \mathrm{cm}^{2}$. The emission measure in the $1-10 \mathrm{keV}$ range was an order of magnitude higher than in the ROSAT range for other novae (see Fig. 1). These authors found constraining evidence that, like for V1974 Cyg, N(H) was decreasing in time and discussed how shocks must have occurred inside the ejecta. They concluded that the characteristics and evolution of the spectrum did not indicate a post-maximum wind colliding with material from pre-maximum wind, but rather a non-smooth outflow with varying velocity, which may certain prove to be a challenge for the models.

5 new novae observed within 2 years from the outburst with Chandra and/or XMM-Newton showed non-atmospheric X-ray emission. For a nova at known LMC distance (LMC 2000, Greiner et al. 2003) a luminosity $\mathrm{L}_{\mathrm{x}} \approx 5 \times 10^{-34} \mathrm{erg} \mathrm{cm}^{-2} \mathrm{~s}^{-1}$ was measured in the $0.2-10 \mathrm{keV} 51$ days after the outburst. This is important because Galactic novae distances are often known with poor precision. The recurrent novae IM Nor and CI Aql were X-ray sources without any indication of emission from the central star (Greiner \& DiStefano 2002, Tepedelenlioglu et al. 2004), and 3 out of 5 novae observed with XMMNewton 2 to 4 years post-outburst showed only non atmospheric emission. For V2487 Oph (1998) the Xray flux is attributed to ongoing accretion (Hernanz \& Sala 2002). My analysis or archival observations of V Sgr 4633 (1998) shows that the X-ray emission is likely to be originated in a slowly cooling shell. I also found that LZ Mus (1998) was not an X-ray source 3 years after outburst, that V4444 Sgr (1999) was only marginally detected, and that V 1141 Sco (1997), if the Beppo-SAX detection was real (see Orio et al. 2001a), must have cooled down and does not emit $X$ rays 3 years later.

An unusual obscuration of the absorption lines spectrum of the WD in nova V4743 Sgr, the main Xray source showing an atmospheric absorption lines spectrum, was recently observed for in V4743 Sgr and revealed the emission like spectrum of a less luminous source (Ness et al. 2003). Like for the last observations of V382 Vel, this second source is most likely of nebular origin (either due to shocks or photoionization by the central source, see Greiner et al. 2003 for a discussion). This observation poses a doubt as to whether we can discriminate between wind and WD atmosphere with a CCD-type detector, when they are superimposed and at a stage of comparable luminosity. For V4743 Sgr we were lucky, because the emission lines source was observed to be much less bright then the central source (Ness et al. 2003), but this is not always so at any epoch for any nova. The evolution of the two sources of soft $\mathrm{X}$-rays, the nebula and the $\mathrm{WD}$, may have a peak in luminosity around the same time. Spatially resolving the shell is not a realistic goal in most cases, because the angular diameter of the shell at typical nova distance $\mathrm{d} \geq 1 \mathrm{kpc}$ is mostly $<1$ arcsec in the first two years. With CCD-like instruments, deriving the WD 
TABLE 1

TIME TO REACH SUPERSOF'T X-RAY

MAXIMUN ( $T_{\text {SOFT }}$ ) AND CONSTANT BOLOMETRIC LUMINOSITY PHASE LENGTH $\left(\mathrm{T}_{\mathrm{BOL}}\right)$ FOR CLASSICAL AND RECURRENT NOVAE

\begin{tabular}{lrr}
\hline NOVA & $\mathrm{T}_{\text {SOFT }}$ & $\mathrm{T}_{\mathrm{BOL}}$ \\
\hline GQ Mus & & $9-10$ years \\
V1974 Cyg & $434-511$ days & $2-3$ years \\
V382 Vel & $59<\mathrm{t} \leq 184$ days & $<265$ days \\
V1494 Aql & $180-210$ days & $2.5-3$ years \\
V4743 Sgr & $120-180$ days & \\
N LMC 1995 & $\geq 7$ months & $6<\mathrm{t}<8$ years \\
N in M31 & $\leq 11$ months & $2-2.5$ years \\
N LMC 2000 & $<51$ days & $<51$ days \\
U Sco & $\simeq 20$ days & \\
IM Nor & $30<\mathrm{t}<150$ days & \\
CI Aql & $<16$ months & $<16$ months \\
\hline
\end{tabular}

parameters may be impossible because we do not resolve in the spectrum the nebular emission lines but only observe a composite "pseudo-continuum".

\section{THE SUPERSOFT CENTRAL X-RAY SOURCE}

In Table 11 show a range of time to reach maximum in supersoft X-rays and the duration of the supersoft X-ray phase (which is also the length of the whole constant bolometric luminosity phase). I have found no clear correlation of the times in the table with the parameters used to classify novae, like $\mathrm{t}(3)$ (the time for a decay by 3 magnitudes in optical). In Greiner et al. (2003) we explain the length of the constant bolometric luminosity phase in terms of the complex interplay between the leftover mass (difference between the mass necessary to ignite the nova flash, which is a function of the WD mass M(WD) and mass transfer rate $\dot{m}$, in turn dependent on the orbital period, and the ejected mass $M(e j))$ and the minimum mass necessary for thermonuclear burning to continue (a function of $\mathrm{M}(\mathrm{WD})$ ). However, $M(W D)$ is normally unknown and be inferred from a theoretical correlation with $\mathrm{t}(3)$, but other factors also effect $t(3) . M(e j)$ is only roughly estimated in the literature, with huge error bars. It is still difficult to predict clearly how often, and why, novae retain part of the accreted envelope or eject instead more material, namely even WD material. In my opinion, the situation is in still confusing and we cannot yet make statistically significantly predictions concerning the end product of nova evolution.
The last three lines of Table 1 show in fact the results known on recurrent novae (RN). U Sco, a very fast $R N$, was observed to be a supersoft X-ray source only 20 days after maximum, and it was not obscrved again (Kahabka et al. 1999). The optical evolution of CI Aql was significantly slower than the one of U Sco, and for this reason Greiner \& DiStefano (2002) proposed to observe it only 16 months after the outburst, expecting an X-ray light curve like V1474 Cyg, however the supersoft X-ray source was not detected. IM Nor, another RN which shares many physical characteristics with CI Aql, was observed with Chandra twice, at much earlier epochs. 1 and 6 months post-outburst. Given t.(3) of the same order of magnitude as CI Aql but a nuuch shorter orbital period (which most likely indicates higher $\dot{\mathrm{m}}$ and lower envelope mass necessary to trigger the outburst), IM Nor was expected to turn into a supersoft $\mathrm{X}$-ray source within only half a year. Optical observations showed that the envelope became optically thin (Tepedelenlioglu et al., 2004, in preparation). However, the result was negative again. Most models foresee that sufficient hydrogen to burn is left over on the WD of RN. RN are thus type Ia SN candidates, because the WD is initially massive, and it grows towards the Chandrasekhar mass after repeated outbursts. However, the non-detection in supersoft $\mathrm{X}$-rays indicates that $\mathrm{RN}$ remain a puzzle. Was CI Aql observed too late and did IM Nor appear as a supersoft X-ray source only for a short time between the two observations? Perhaps we will have to conclude that the WD mass does not grow, but more statistics and more frequent sampling of $\mathrm{RN}$ light curves are necessary.

V1974 Cyg, observed with ROSAT in 1992-1993, proved that Galactic novae can be the brightest supersoft X-ray sources, and actually some of the brightest among all X-ray sources. Two novae observed with ROSAT and other satellites as supersoft X-ray sources for longer than others: GQ Nus and N LMC 1995. N LMC 1995 was observed again with $X M M-N e w t o n$ and still observed as a supersoft X-ray source 6 years after the outburst (Orio et al. 2003). Atmospheric models did fit the observed X-ray spectrum, apparently not obscured any more, even partially, by nebular emission. However, this nova was not sufficiently bright for grating spectra, so important parameters remained unconstrained. A recent follow up observation with XMM-New'ton (Orio et al. 2004, in preparation) showed that the supersoft X-ray source however almost faded after \& years.

We still have only small number statistics, but TOO observation done with Chandra by a large 
international collaboration indicate that probably about half of all novae turn into super-bright sources, at least as luminous as V1974 Cyg, for a few months. We do not know whether the supersoft X-ray source phase is extremely short lived in other novae or does not occur. The light curves of V382 Vel, V1494 Aql and V4743 Sgr show intense variability that is not clearly understood. For V382 Vel, there were short obscurations (Orio et al. 2002). In the case of V1494 Aql a flare was observed, which lasted for about 20 minutes. The count rate increased by a factor of 10 . V4743 Sgr (see also Krautter 2003, this conference) a bright nova that reached $V(\max )=5$ on September 20 2002, was observed in X-rays in November of 2002 (with Chandra ACIS-S), on March 202003 (with Chandra LETG+HRC), in April 42003 (with $X M M-N e w t o n)$, and in June and September of 2003 (again with Chandra LETG+HRC.) The nova had flared up by March 2003: at the time of the second observation, for 3.6 hours the count rate was an astonishing $40 \mathrm{cts} \mathrm{s}^{-1}$ with the LETG, until the obscuration mentioned in Section 3 started. This dramatic fading was no longer detected in April, July and September, although it April the observation was longer than the optical period, so an eclipse seems to be ruled out.

The most interesting discovery in the nova $X$ ray light curves are the non-radial oscillations of the WD, first proposed to explain the power spectrum of V1494 Aql (Drake et al. 2002) and then observed, I dare say beyond doubts, for V4743 Sgr. In Leibowitz et al. (2003), we discuss the power spectrum of this nova. The two highest peaks correspond to the nearby periodicities 1308 and $1374 \mathrm{~s}$, respectively. A $1325 \mathrm{~s}$ period detected in the March Chandra light curve (Ness et al. 2003), may be due to the interference of these two periods. While the $1308 \mathrm{~s}$ period has a sinusoidal structure, the $1374 \mathrm{~s}$ is not symmetric. Its first overtone also appears in the power spectrum, but it is energy dependent and almost disappears in the light curve is extracted in the energy range $0.2-0.4 \mathrm{keV}$ (see Fig. 7 in Leibowitz et al. 2003). In addition there are many more periodicities, as it is true for pulsating PG1059 stars and WD in general. The rich power spectrum, shown in Fig. 4 in Leibowitz et al. (2003), appeared to change somewhat in the subsequent observations in the summer of 2003.

Blue-shifted absorption features corresponding to high ionization states appear in both the Chandra and $X M M-N e w t o n$ spectra. While the velocity was $\simeq 2400 \mathrm{~km} / \mathrm{s}$ in March spectrum, absorption features with lower velocity are also identified in the April
$X M M-N e w t o n$ spectrum. The blue shift seems to indicate that the nova wind starts at the very base of the WD atmosphere. In addition to the absorption features, some of the emission features due to the nebula can be distinguished as they are superimposed on the atmospheric spectrum. A preliminary analysis of the XMM-RGS spectra of April of 2003 indicates that carbon was not very abundant, that the effective temperature was $\mathrm{T} \approx 450000 \mathrm{~K}$, and that effective gravity was $\mathrm{g} \leq 10^{8} \mathrm{~cm} \mathrm{~s}^{-2}$.

The non radial WD pulsations may prove to be an additional and important way to derive WD parameters. If we succeed in developing detailed models, these oscillations, which are easily detectable in the X-ray light curve, are a promising possibility to probe $\mathrm{T}, \mathrm{g}$ and the abundances of the WD, other than by using WD atmospheric models, thus yielding independent means to derive these parameters.

\section{REFERENCES}

Anupama, G.C., \& Dewangan, G.C. 2000, AJ, 119, 1359

Balman, S., Krautter, J., \& Ögelman H., 1998, ApJ, 499, 395

Burwitz V., Starrfield, S., Krautter, J. \& Ness, J-U. 2002, in Classical Nova Explosions, AIP Conf. Proc. Vol. 637, M. Hernanz \& J. Jose eds., p. 377

Contini M., Orio M., \& Prialnik D. 1995, MNRAS, 275, 195

Drake, J., et al. 2003, ApJ, 584, 448

Greiner, J. 2000, New Astronomy, 5/3, 137

Greiner, J., \& DiStefano, R. 2002, ApJ, 578, L59

Greiner, J., Orio, M., \& Schartel, N. 2003, A\&A, 405, 703

Hernanz, M. \& Sala, G. 2002, Science, 298, 393

Kahabka, P., Hartmann, H.W., Parmar, A.N., \& Neguerela, I. 1999, A\&A, 347, L43

Iijima, T. 2002, A\&A, 387, 1013

Leibowitz, E., Orio, M., et al. 2003, preprint

Livio, M., et al. 1992, ApJ, 394, 217

Lloyd, H.M., et al. 1992, Nature, 356, L222

Mason, K.O., Cordova, F.A., Bode, M.F., \& Barr, P. 1985, in: RS Oph (1985) and the Recurrent Nova Phenomenon, ed. M.F. Bode, VNU Science Press (Utrecht), 167

Mukai, K., \& Ishida, M. 2001, ApJ, 501, 1024

Ness J-U., et al. 2003, ApJ, 594, L127

Ögelman, H., Krautter, J. \& Beuermann, K. 1984, 287, L31

Ögelman, H., Krautter, I. \& Beuermann, K. 1987, A\&A, 177,110

Orio, M., Covington, J., \& Ögelman, H. 2001a, 373, 542

Orio, M., et al. 1996, ApJ, 463, 221

Orio, M., et al. 2001b, MNRAS, 326, L13

Orio, M., et al. 2002, MNRAS, 333, 111

Orio, M., et al. 2003, ApJ, 594, 435

Tepedelenlioglu, E., Orio, M. Starrfield, S., et al. 2004, in preparation 